\title{
Technologies for highly granular electromagnetic and hadronic calorimeters
}

\author{
Huong Lan Tran*i \\ Deutsches Elektronen-Synchrotron (DESY), Hamburg, Germany \\ E-mail: huong.lan.tranedesy.de
}

\begin{abstract}
At future $e^{+} e^{-}$machines, many of the interesting physics processes result in multi-jet final states, with jets coming from either $\mathrm{W}$ or $\mathrm{Z}$ boson decay. It is therefore desired to discriminate $\mathrm{W}$ and $\mathrm{Z}$ hadronic decays, which is largely driven by the jet energy resolution. A W/Z separation of 2.3 $2.6 \sigma$ leads to the requirement of a jet energy resolution of 3-4\% over a wide range of jet energies. This can be achieved with the Particle Flow reconstruction approach, in which the separation of charged and neutral particles is of substantial role. Highly granular calorimeters placed inside a magnetic coil is therefore desired. The CALICE collaboration has been developing various electromagnetic and hadronic calorimeter concepts to meet these requirements. As a first step, the physics prototypes were built to prove the capability to deliver the desired physics. As a next step, technological prototypes have to be built to demonstrate the feasibility of building such detectors in reality. In this talk/proceeding, we discuss briefly the results obtained with the physics prototypes, followed by the recent developments of the technological prototypes.
\end{abstract}

38th International Conference on High Energy Physics

3-10 August 2016

Chicago, USA

* Speaker.

${ }^{\dagger}$ on behalf of the CALICE collaboration 


\section{Motivation for highly granular calorimeters}

Over the last decade, the particle physics community has intensively discussed about the possibility of building a new linear $e^{+} e^{-}$collider. The new machine will not just provide means for new physics discovery but also for precision measurements. Many of the interesting physics processes result in multi-jet final states, with jets coming from either $\mathrm{W}$ or $\mathrm{Z}$ boson decay. It is therefore desired to discriminate $\mathrm{W}$ and $\mathrm{Z}$ hadronic decays. Whether or not this is achievable depends strongly on the jet energy resolution of the detector. A W/Z separation of $2.3-2.6 \sigma$ leads to the requirement of a jet energy resolution of 3-4 \% over a wide range of jet energies. This cannot be attained with the traditional calorimetric approach where all the jet components are fully measured in the electromagnetic and hadronic calorimeters (ECAL/HCAL). In the particle flow (PFlow) approach, only the energies of neutral particles are measured with the calorimeters: neutral hadrons in HCAL and photons in ECAL. For charged particles, the energy is much more precisely measured in the tracker. Since in typical jets, $60 \%$ of the jet energy are carried by charged hadrons, $30 \%$ by photons and by $10 \%$ from neutral hadrons, a better jet energy resolution can be reached with the PFlow approach.

For an optimal performance of the PFlow reconstruction approach, the detector design should be adapted: highly granular calorimeters placed inside a magnetic coil, a tracker providing very good momentum resolution with low material budget, a high B field and very good particle identification (ParticleID). In this approach, the calorimeters play a substantial role: they strongly determine the ability to distinguish photons from electrons in the ECAL and neutral hadrons from charged hadrons in the HCAL [1]. To achieve the best distinction, the calorimeters need to have high granularity in both transversal and longitudinal direction. Such designs have been long developed and tested by the CALICE collaboration.

\section{The CALICE collaboration and its prototypes}

The CALICE collaboration with 336 physicists/engineers from 57 institutes has successfully developed several concepts of highly granular electromagnetic and hadronic calorimeters. Different active material options have been investigated.

- Silicon: The silicon characteristic gives the advantage of building a compact detector with minimal thickness. The material itself is not sensitive to ambient changes and provides stable channel-to-channel calibration.

- Scintillator: Scintillator has long been the ideal choice of sensor for calorimetry. It is robust, reliable and easy to machine which gives the flexibility to choose the cell size and thickness. Therefore, compared to silicon, scintillator can provide a higher sampling fraction. Scintillating light can be read out with Silicon Photomultipliers (SiPM). The new generation of industrially available SiPMs has drastically improved quality which allows a noise free operation.

- Gaseous: Gaseous detectors can be easily segmented and therefore they have potentially better imaging capability. The deposited energy in gas, however, is affected by large fluctuations. The effect of fluctuation on energy reconstruction can be eliminated with the hit 
counting approach. With one bit readout (digital), up to a certain energy where the average number of hit per cell is close to one, the energy can be reconstructed with good accuracy. Above this range, the digital response is no longer linear. As a result, the concept of two bit readout (semi-digital) is developed to restore the linearity and resolution. One very good and well-known technology for gaseous detector is Glass Resistive Plate Chamber (GRPC).

To prove the physics potential of these technologies, various prototypes have been built by the CALICE collaboration and intensively tested in different beam facilities. The successful results from these physics prototypes have validated the physics potential of the detector concepts. However, to demonstrate the feasibility of building such detectors in reality, technological prototypes have to be built. These prototypes must be functioning with fully integrated electronics and be scalable to a full detector. The mass production aspect also needs to be established, as the construction of a large detector is timely constraint. In the following section, the results obtained with the physics prototypes will be discussed, followed by the most recent updates on the technological prototype status.

\section{Electromagnetic calorimeters}

\subsection{Silicon ECAL}

The Silicon ECAL physics prototype is composed of 30 layers of silicon wafers and tungsten absorber. The silicon wafers are of $525 \mu \mathrm{m}$ thin and segmented into $1 \times 1 \mathrm{~cm}^{2}$ cell size. The prototype has been exposed to electron and positron beams in the energy range of $6-45 \mathrm{GeV}$. The calorimeter response is linear to within approximately $1 \%$. The energy resolution has a stochastic term of $(16.6 \pm 0.1) \% / \sqrt{E(G e V)}$, whereas the constant term is (1.1 \pm 0.1$) \%$ (Fig. 1a) [2].

For the technological prototype, new silicon sensors are studied which have now an improved guard ring design for a reduced dead zone at the edge of each sensor and a decreased capacitative cross-talk between the guard ring and the pixels. The cell size is reduced to $0.5 \times 0.5 \mathrm{~cm}^{2}$. Four boards with fully integrated electronics have been consecutively connected to test the power and clock distribution (Fig. 1b). With these same boards, a prototype of 4-layer has been built and tested at CERN in 2015. The prototype showed very smooth running. A common beam test with the Semi-Digital HCAL (SDHCAL) (section 4.1) took place in June 2016 at CERN with 10 layers of ECAL and the full SDHCAL.

\subsection{Scintillator ECAL}

The scintillator ECAL prototype is a sandwich of 30 layers of scintillator active layers and tungsten absorber. The active layers are composed of strips of $1 \times 4.5 \mathrm{~cm}^{2}$ size and $3 \mathrm{~mm}$ thickness. The orientation of the strips in consecutive layers are orthogonal, which makes an effective granularity of $1 \times 1 \mathrm{~cm}^{2}$. Each scintillator strip is read out by a SiPM. The prototype has been exposed to electron beams in the energy range of $1-32 \mathrm{GeV}$ at the Fermilab beam test facility. A good resolution has been achieved with a stochastic term of $12.8 \pm 0.1$ (stat.) \pm 0.4 (syst.) $\%$ and a constant term of 1.0 \pm 0.1 (stat.)+0.5-1.0(syst.)\% (Fig. 2a) [3].

New versions of scintillator strips and SiPMs have been investigated. The new strips of $0.5 \times 4.5 \mathrm{~cm}^{2}$ size gives an effective granularity of $0.5 \times 0.5 \mathrm{~cm}^{2}$ which improves the imaging ca- 


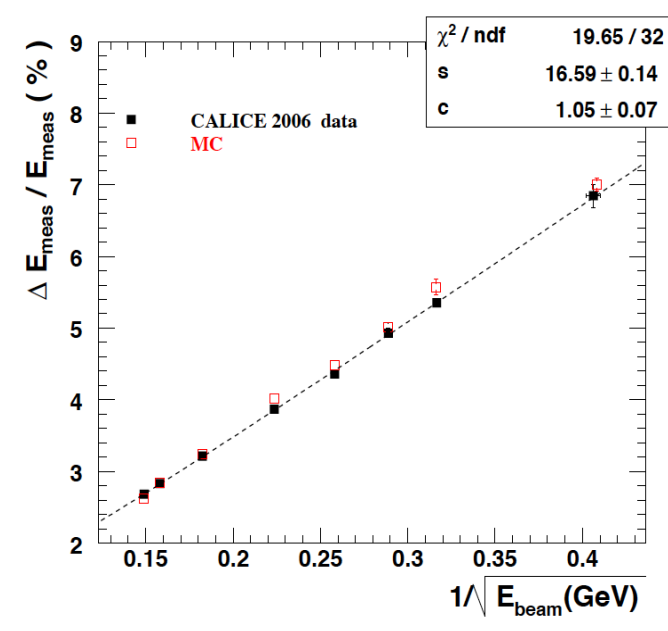

(a) Relative energy resolution ( $\left.\triangle E_{\text {meas }} / E_{\text {meas }}\right)$ as a function of the beam energy obtained with the SiliconTungsten ECAL physics prototype (solid squares), and its usual parametrisation as $s / \sqrt{E} \oplus c$. The values expected from simulation are superposed (open squares).

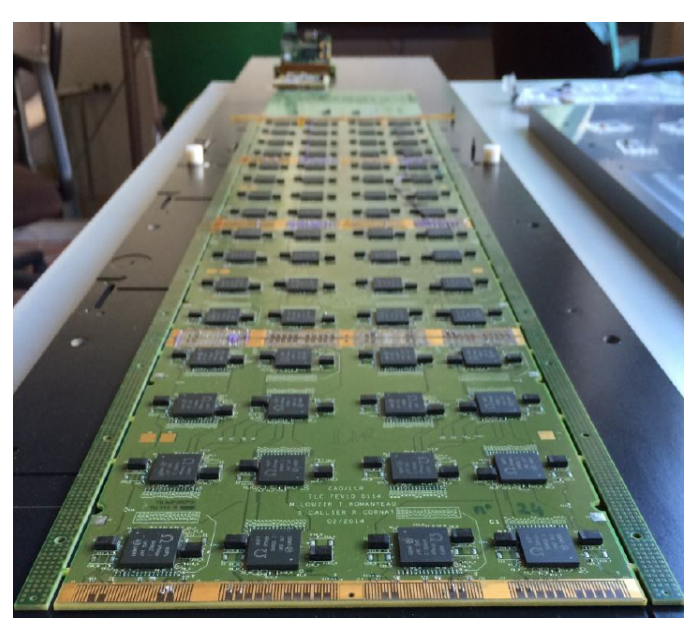

(b) Silicon ECAL longslab with 4 boards.

Figure 1: Towards a full Silicon-Tungsten ECAL technological prototype.

pacity. The readout SiPMs are placed at the bottom of the strips to reduce dead space. A new generation of SiPMs with 10000 pixels are used for a much wider dynamic range.

Three ECAL Base Units (EBU) with latest hardware designs have been put in combined test beam with the Scintillator HCAL (section 4.2). The prototype worked smoothly using common Data Acquisition (DAQ). The Strip Splitting Algorithm (SSA) [4] has been applied to the ECAL data to extract fine granularity information from the long strip geometry. The obtained result is very encouraging (Fig. 2b).

\section{Hadronic calorimeters}

\subsection{Gaseous HCAL}

The SDHCAL technological prototype uses RPCs of $1 \times 1 \mathrm{~cm}^{2}$ size, readout boards on only one side of the cassettes (thanks to flexible connections between ASIC boards) and self-triggered and power-pulsed front-end chips (HARDROC) [5]. It has two bit readout to mitigate the effect of saturation. Consisting of $50 \mathrm{RPC}$ cassettes inserted inside a $1 \mathrm{~m}^{3}$ steel absorber structure, the prototype was tested at CERN in 2012 in auto-triggering mode and with power pulsing using SPS spill structure for power consumption reduction. With data collected in electron and pion beams of 1-100 GeV (Fig. 3a), it has demonstrated a significant improvement of pion resolution compared to the one bit readout scheme in the energy range above $30 \mathrm{GeV}$ (Fig. 3b).

One of the challenges for the SDHCAL is to demonstrate the capability to build the biggest layer of a module of which the design is inspired by the International Large Detector (ILD) concept. The construction of 1-layer prototype has been planned with newest generation electronics and new Detector Interface (DIF) which handles up to 432 readout chips in one layer (compared to 


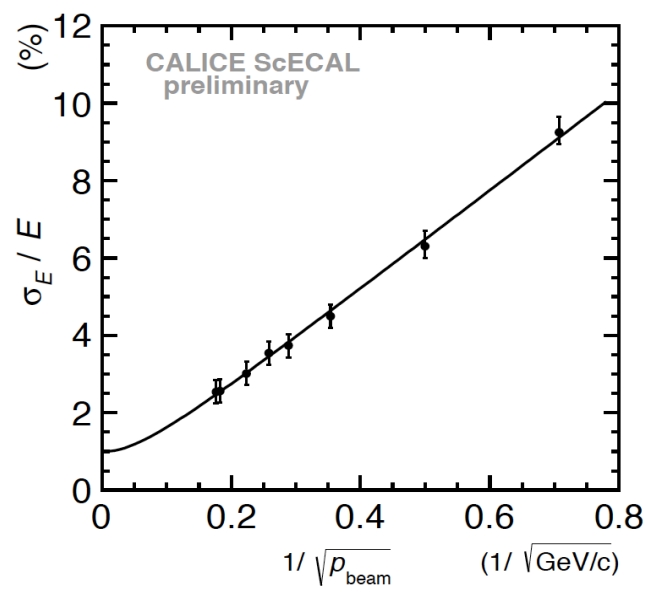

(a) The energy resolution of ScECAL as a function of the inverse of the square root of the beam momentum.

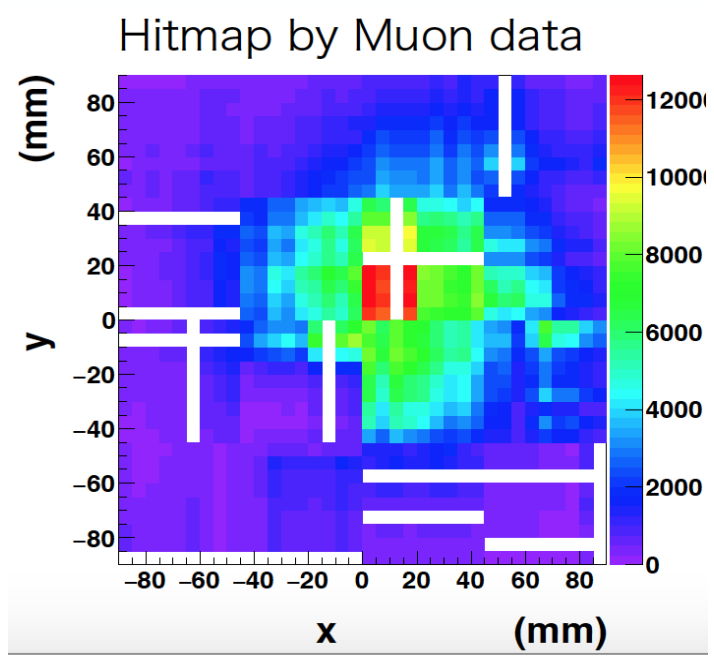

(b) ECAL hit map reconstructed using strip splitting algorithm.

Figure 2: Results from Scintillator ECAL physics prototype (left) and from the combined beam test of scintillator ECAL and HCAL (right).

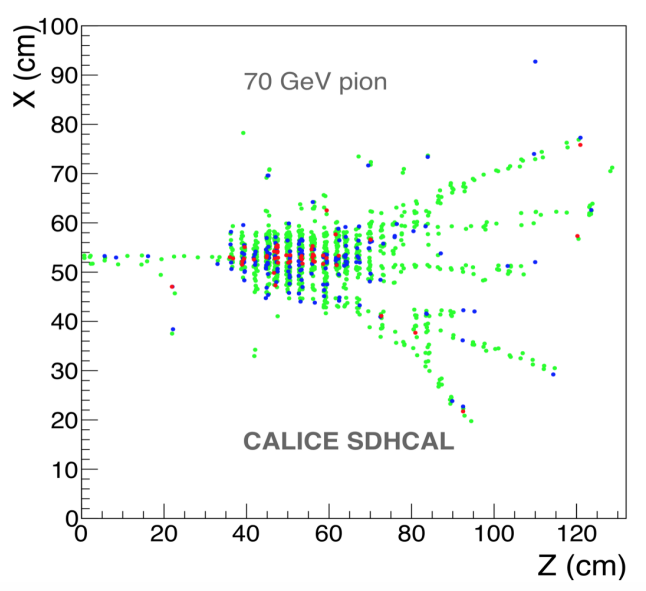

(a) A $70 \mathrm{GeV}$ pion even occurred in the SDHCAL which shows hits in different colours corresponding to three different thresholds.

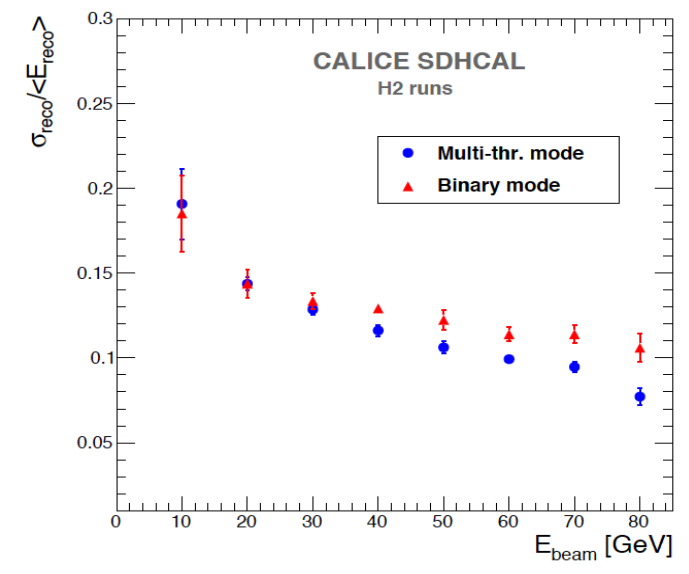

(b) Pion resolution obtained with multi-threshold and binary mode in the energy range of $10-80 \mathrm{GeV}$.

Figure 3: Results from the SDHCAL technological prototype. 


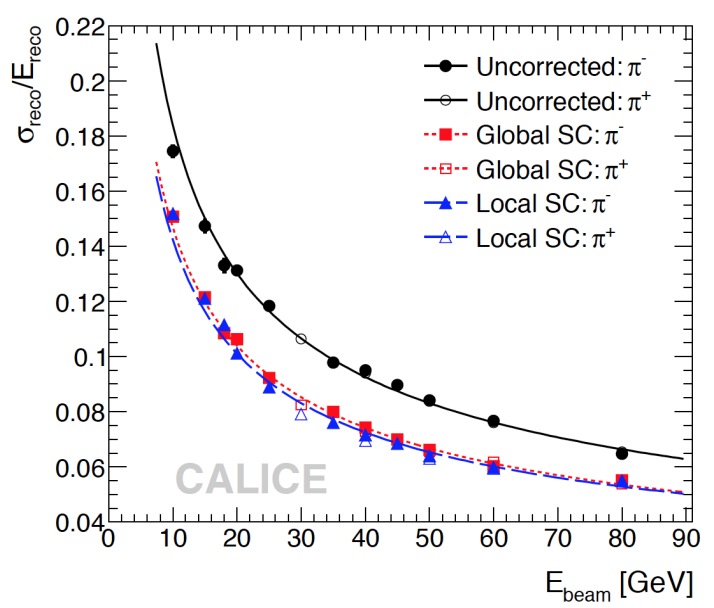

(a) Pion resolution is significantly improved with the software compensation techniques.

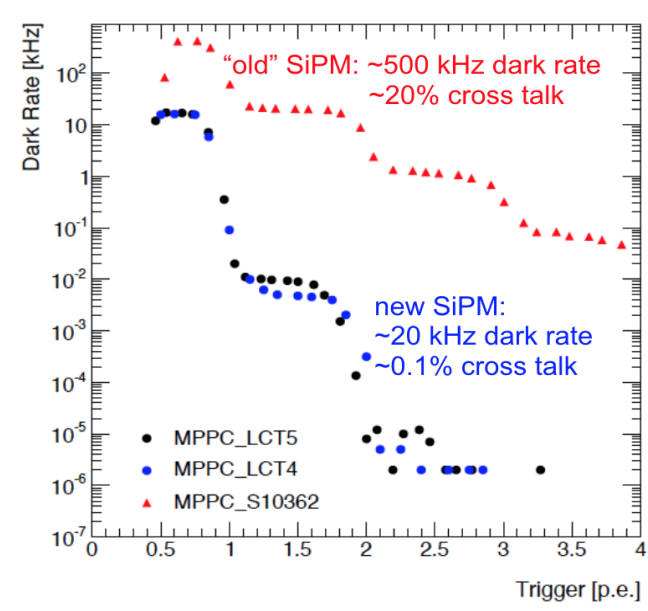

(b) Dark count rates of previous generation Hamamatsu SiPMs (red) and new devices (blue, black).

Figure 4: Towards a full AHCAL technological prototype.

48 readout chips in previous version). It is also planned to have beam tests with the ILD-inspired mechanical structure.

\subsection{Scintillator HCAL}

The Scintillator HCAL is a $1 \mathrm{~m}^{3}$ sandwich detector of 38 layers of absorber plates (steel or tungsten) and scintillator tiles of $5 \mathrm{~mm}$ thickness. The size of the tiles varies from $3 \times 3 \mathrm{~cm}^{2}$ (in the most central part) to $12 \times 12 \mathrm{~cm}^{2}$ (most outer part). The scintillating light is read out by SiPMs and the output is digitised with 16 bit Analogue to Digital Converter (ADC), which provides precise measurement of the deposited energy. The concept is therefore called Analogue HCAL (AHCAL). Beam tests combining the AHCAL, the silicon ECAL (section 3.1) and a tail catcher were intensively conducted from 2006 to 2012 and have demonstrated the capability to deliver the desired physics. Most importantly, by applying the powerful software compensation techniques based on the energy density, the shower stochastic fluctuations has been reduced from $58 \% / \sqrt{E}$ to $45 \% / \sqrt{E}$ while the constant term is kept smaller than $2 \%$ (Fig. 4a) [7]. Offline overlay of different events were also used to assess key aspects of the PFlow in a realistic detector configuration. The agreement between the PFlow performance achieved with real calorimeter prototype data and with the MC simulation demonstrates that the extrapolation to the complete detector is reliable [8].

Recently, the AHCAL group has started to build a technological prototype which is scalable for a full realistic detector. In this design, the prototype with fully integrated electronics can function in both auto-trigger and external trigger mode. The power pulsing functionality reduces significantly the power consumption. In order to prove the capability of building a full detector, the design of the detector components are also improved such that they can be produced and assembled automatically. Surface mounted SiPMs with the corresponding tile design allows an automated pick-and-place assembly which is now used routinely in the AHCAL group. The quality of the new generation of industrially available SiPMs is also drastically improved over the past years (Fig. 4b) allowing a much more stable operation and simplified calibration procedure. 


\section{Applications to LHC experiments}

Inspired by the success of CALICE highly granular calorimeters, the High Granular Calorimeter (HGCAL) upgrade of the CMS end-cap calorimeter for the high luminosity phase of the LHC strongly involves the CALICE-developed technologies. It is foreseen to equip the front and innermost sections with silicon sensors, similar to the ECALs envisaged for the linear collider detectors, while for the rear and outer sections a scintillator and SiPM based readout is conceived.

\section{Acknowledgements}

This project has received funding from the European Union's Horizon 2020 Research and Innovation programme under Grant Agreement no. 654168.

\section{References}

[1] Thomson, M.A. Particle Flow Calorimetry and the PandoraPFA Algorithm, 10.1016/j.nima.2009.09.009 Nucl.Instrum.Meth.

[2] CALICE Collaboration. Response of the CALICE Si-W Electromagnetic Calorimeter Physics Prototype to Electrons, NIM A608 (2009) 372; e-print: arXiv:0811.2354.

[3] CALICE Collaboration. First Stage Analysis of the Energy Response and Resolution of the Scintillator ECAL in the Beam Test at FNAL, 2008, CALICE Note CAN-016, https://twiki.cern.ch/twiki/pub/CALICE/CaliceAnalysisNotes/CAN-016.pdf.

[4] K. Kotera et al. A novel strip energy splitting algorithm for the fine granular readout of a scintillator strip electromagnetic calorimeter, Nucl.Instrum.Meth. A789 (2015) 158-164.

[5] I. Laktineh. Development of a semi-digital hadronic calorimeter using GRPCs for future linear collider experiments, Nucl. Instr. and Meth. A 628 (2011) 328.

[6] CALICE Collaboration. First results of the CALICE SDHCAL Technological Prototype, CALICE Analysis Note CAN-037, https://twiki.cern.ch/twiki/pub/CALICE/CaliceAnalysisNotes/CAN-037.pdf, 2012.

[7] CALICE Collaboration. Hadronic energy resolution of a highly granular scintillator-steel calorimeter using software compensation techniques, JINST 7 (2012) P09017.

[8] CALICE Collaboration Tests of a particle flow algorithm with CALICE test beam data, JINST 6 (2011) P07005. 